\title{
Generalized modular fractal spaces and fixed point theorems
}

\author{
Alireza Alihajimohammad ${ }^{1 *}$ and Reza Saadati ${ }^{2^{*}}$ (D)
}

"Correspondence:

a.alihajimohammadi@srbiau.ac.ir;

rsaadati@eml.cc; rsaadati@iust.ac.ir

1 Department of Mathematics,

Science and Research Branch,

Islamic Azad University, Tehran, Iran

${ }^{2}$ School of Mathematics, Iran

University of Science and

Technology, Narmak, Tehran, Iran

\begin{abstract}
In this article, we introduce a new concept of Hausdorff distance through generalized modular metric on nonempty compact subsets and study some topological properties of it. This concept with contraction theory and the iterated function system (IFS) helps us to define a generalized modular fractal space.
\end{abstract}

Keywords: Modular metric spaces; Hausdorff distance; Fixed points; Modular fractal space

\section{Introduction}

Chistyakov [6-9] rectified in absorbing manner the structure of a metric modular space and introduced a first countable and Hausdorff topology on it which is very popular in contemporary research these days.

Now, we study the concept of the Hausdorff distance of a given generalized metric modular space on nonempty compact subsets. As an application, we use the concept of contraction and the iterated function system (IFS) on a generalized metric modular space to define a new concept of modular fractal spaces and prove an interesting fixed point theorem in these spaces $[1,2,7,8,10,11]$.

\section{Basic notions and preliminaries}

Now, we recall some notions and basic concepts. Here, we let $\mathbb{I}=[0,1], \mathbb{I}^{0}=(0,1), \mathbb{J}=$ $[0, \infty]$, and $\mathbb{J}^{0}=(0, \infty)$. Let $S$ be a nonempty set. A function $\theta: S \times S \times \mathbb{J}^{0} \rightarrow \mathbb{J}$ is said to be a metric modular (in short MM) on $S$ if it satisfies the following three axioms:

(i) Given $u, v \in X, \theta_{\lambda}(u, v)=0$ for all $\lambda>0$ if and only if $u=v$;

(ii) $\theta_{\lambda}(u, v)=\theta_{\lambda}(v, u)$ for all $\lambda>0$ and $u, v \in S$;

(iii) $\theta_{\lambda+\mu}(u, v) \leq \theta_{\lambda}(u, w)+\theta_{\mu}(w, v)$ for all $\lambda, \mu>0$ and $u, v, w \in S$. Also, the ordered pair $(S, \theta)$ is said to be an MM-space.

Consider a mapping $\Upsilon: S^{3} \times \mathbb{J}^{0} \rightarrow \mathbb{J}$, given by $\Upsilon_{\sigma}(s, t, u)=\Upsilon(s, t, u, \sigma)$, in which $\sigma \in \mathbb{J}^{0}$ and $s, t, u \in S$. In this paper, we consider a generalized metric space in the sense of Chistyakov and introduce the concept of generalized modular metric space (in short, GMM-space) as follows.

(c) The Author(s) 2021. This article is licensed under a Creative Commons Attribution 4.0 International License, which permits use sharing, adaptation, distribution and reproduction in any medium or format, as long as you give appropriate credit to the original author(s) and the source, provide a link to the Creative Commons licence, and indicate if changes were made. The images or other third party material in this article are included in the article's Creative Commons licence, unless indicated otherwise in a credit line to the material. If material is not included in the article's Creative Commons licence and your intended use is not permitted by statutory regulation or exceeds the permitted use, you will need to obtain permission directly from the copyright holder. To view a copy of this licence, visit http://creativecommons.org/licenses/by/4.0/. 
Definition 2.1 ([3]) Let $S$ be a nonempty set. A function $\Upsilon: S \times S \times S \times \mathbb{J}^{0} \rightarrow \mathbb{J}$ is said to be a generalized modular metric on $S$ (in short GMM) if it satisfies the following five axioms:

(GMM-1) $\Upsilon_{\sigma}(s, s, u) \in \mathbb{J}^{0}$ for all $s, u \in S$ and $\sigma \in \mathbb{J}^{0}$ with $s \neq u$.

(GMM-2) $\Upsilon_{\sigma}(s, t, u)=0$ for all $s, t, u \in S$ and $\sigma \in \mathbb{J}^{0}$ if $s=t=u$.

(GMM-3) $\Upsilon_{\sigma}(s, s, u) \leq \Upsilon_{\sigma}(s, t, u)$ for all $s, t, u \in S$ and $\sigma \in \mathbb{J}^{0}$ with $t \neq u$.

(GMM-4) $\Upsilon_{\sigma}(s, t, u)=\Upsilon_{\sigma}(s, u, t)=\Upsilon_{\sigma}(u, s, t)=\cdots$ for all $\sigma \in \mathbb{J}^{0}$.

(GMM-5) $\Upsilon_{\sigma+\delta}(s, t, u) \leq \Upsilon_{\sigma}(s, v, v)+\Upsilon_{\delta}(v, t, u)$ for all $s, t, u \in S$ and $\sigma, \delta \in \mathbb{J}^{0}$. Also the ordered pair $(S, \Upsilon)$ is said to be a GMM-space.

Definition 2.2 ([3]) Let us fix an arbitrary element $s_{0} \in S$ and set $S_{\Upsilon}=\left\{t \in S ; \lim _{\sigma \rightarrow 0} \Upsilon_{\sigma}\left(s_{0}\right.\right.$, $t, u)=0$ for some $u \in S\}$. The set $S_{\Upsilon}$ is called a modular set.

Proposition 2.3 ([3]) Let $(S, \Upsilon)$ be a GMM-space, for any s, $t, u, v \in S$ it follows that

(1) If $\Upsilon_{\sigma}(s, t, u)=0$ for all $\sigma>0$, then $s=t=u$.

(2) $\Upsilon_{\sigma}(s, t, u) \leq \Upsilon_{\frac{\sigma}{2}}(s, s, t)+\Upsilon_{\frac{\sigma}{2}}(s, s, u)$ for all $\sigma>0$.

(3) $\Upsilon_{\sigma}(s, t, t) \leq 2 \Upsilon_{\frac{\sigma}{2}}(s, s, t)$ for all $\sigma>0$.

(4) $\Upsilon_{\sigma}(s, t, u) \leq \Upsilon_{\frac{\sigma}{2}}(s, v, u)+\Upsilon_{\frac{\sigma}{2}}(v, t, u)$ for all $\sigma>0$.

(5) $\Upsilon_{\sigma}(s, t, u) \leq \frac{2}{3}\left(\Upsilon_{\frac{\sigma}{2}}(s, t, v)+\Upsilon_{\frac{\sigma}{2}}(s, v, u)+\Upsilon_{\frac{\sigma}{2}}(v, t, u)\right)$ for all $\sigma>0$.

(6) $\Upsilon_{\sigma}(s, t, u) \leq\left(\Upsilon_{\frac{\sigma}{2}}(s, u, v)+\Upsilon_{\frac{\sigma}{4}}(t, v, v)+\Upsilon_{\frac{\sigma}{4}}(u, v, v)\right)$ for all $\sigma>0$.

If $(S, \theta)$ is an MM-space, then $(S, \theta)$ can define a GMM-space on $S$ by

$\left(E_{x}\right) \Upsilon_{\sigma}^{x}(s, t, u)=\frac{1}{3}\left\{\theta_{\sigma}(s, t)+\theta_{\sigma}(t, u)+\theta_{\sigma}(s, u)\right\}$,

$\left(E_{m}\right) \Upsilon_{\sigma}^{m}(s, t, u)=\max \left\{\theta_{\sigma}(s, t)+\theta_{\sigma}(t, u)+\theta_{\sigma}(s, u)\right\}$ for all $\sigma>0$.

We showed that a modular metric (MM) can introduce a generalized modular metric (GMM). Now, we study the converse, consider the GMM $\Upsilon_{\sigma}$ on $S$, then $\left(E_{\theta}\right) \theta_{\sigma}^{\Upsilon}(s, t)=$ $\Upsilon_{\sigma}(s, t, t)+\Upsilon_{\sigma}(s, s, t)$ defines a modular metric MM on $S$ for all $\sigma \in \mathbb{J}^{0}$. Also, there are the following relationships among $\Upsilon_{\sigma}$, $\Upsilon_{\sigma}^{x}$, and $\Upsilon_{\sigma}^{m}$ :

$$
\Upsilon_{\sigma}(s, t, u) \leq \Upsilon_{\sigma}^{x}(s, t, u) \leq 2 \Upsilon_{\sigma}(s, t, u)
$$

and

$$
\frac{1}{2} \Upsilon_{\sigma}(s, t, u) \leq \Upsilon_{\sigma}^{m}(s, t, u) \leq 2 \Upsilon_{\sigma}(s, t, u)
$$

for all $\sigma>0$.

By the following equalities we can find the relationships among modular metrics induced by different GMMs. For $s, t \in S$, it is easy to show $\theta_{\sigma}^{\Upsilon^{x}}(s, t)=\frac{4}{3} \theta_{\sigma}(s, t)$ and $\theta_{\sigma}^{\Upsilon^{m}}(s, t)=$ $2 \theta_{\sigma}(s, t)$ for all $\sigma>0$.

Definition 2.4 ([3]) Let $(S, \Upsilon)$ be a GMM-space. Then, for $s_{0} \in S_{\Upsilon}$ and $\varepsilon>0$, the $\Upsilon$-ball with center $s_{0}$ and radius $\varepsilon$ is

$$
B_{\Upsilon}\left(s_{0}, \varepsilon\right)=\left\{t \in S_{\Upsilon}: \Upsilon_{\sigma}\left(s_{0}, t, t\right)<\varepsilon \text { for all } \sigma>0\right\}
$$

Proposition 2.5 ([3]) Let $(S, \Upsilon)$ be a GMM-space. Then, for any $s_{0} \in S_{\Upsilon}$ and $\varepsilon>0$, we have

(i) if $\Upsilon_{\sigma}\left(s_{0}, s, t\right)<\varepsilon$ for all $\sigma>0$, then $s, t \in B_{\Upsilon}\left(s_{0}, \varepsilon\right)$.

(ii) if $t \in B_{\Upsilon}\left(s_{0}, \varepsilon\right)$, then we can find $\delta>0$ such that $B_{\Upsilon}(t, \delta) \subseteq B_{\Upsilon}\left(s_{0}, \varepsilon\right)$. 
From Proposition 2.5 we can conclude that the family of all $\Upsilon$-balls $\Gamma=\left\{B_{\Upsilon}(s, \varepsilon) \mid s \in\right.$ $S, \varepsilon>0\}$ is the base of a topology $\mathcal{T}\left(\Upsilon_{\sigma}\right)$ on $S_{\Upsilon}$.

Definition 2.6 ([3]) Let $(S, \Upsilon)$ be a GMM-space. The sequence $\left\{s_{n}\right\}_{n \in \mathbb{N}} \subseteq S_{\Upsilon}$ is $\Upsilon$ convergent to $s$ if it converges to $s$ in the topology $\mathcal{T}\left(\Upsilon_{\sigma}\right)$.

Proposition 2.7 ([3]) Let $(S, \Upsilon)$ be a GMM-space and $\left\{s_{n}\right\}_{n \in \mathbb{N}} \subseteq S_{\Upsilon}$.

Then the following are equivalent:

(1) $\left\{s_{n}\right\}_{n \in \mathbb{N}}$ is $\Upsilon$-convergent to $s$;

(2) $\theta_{\sigma}^{\Upsilon}\left(s_{n}, s\right) \rightarrow 0$ as $n \rightarrow \infty$, i.e., $\left\{s_{n}\right\}$ converges to s relative to the $M M \theta_{\sigma}^{\Upsilon}$;

(3) $\Upsilon_{\sigma}\left(s_{n}, s_{n}, s\right) \rightarrow 0$ as $n \rightarrow \infty$ for all $\sigma>0$;

(4) $\Upsilon_{\sigma}\left(s_{n}, s, s\right) \rightarrow 0$ as $n \rightarrow \infty$ for all $\sigma>0$;

(5) $\Upsilon_{\sigma}\left(s_{m}, s_{n}, s\right) \rightarrow 0$ as $m, n \rightarrow \infty$ for all $\sigma>0$.

Definition 2.8 ([3]) Let $(S, \Upsilon)$ be a GMM-space. Then $\left\{s_{n}\right\}_{n \in \mathbb{N}} \subseteq S_{\Upsilon}$ is called $\Upsilon$-Cauchy sequence if, for every $\varepsilon>0$, we can find $N_{\varepsilon} \in \mathbb{N}$ such that $\Upsilon_{\sigma}\left(s_{n}, s_{m}, s_{q}\right)<\varepsilon$ for all $n, m, q \geq$ $N_{\varepsilon}$ and $\sigma>0$.

A GMM-space $S$ is called $\Upsilon$-complete if every $\Upsilon$-Cauchy sequence in $S$ is a $\Upsilon$ convergent sequence in $S$.

Proposition 2.9 ([3]) Let $(S, \Upsilon)$ be a GMM-space and $\left\{s_{n}\right\}_{n \in \mathbb{N}} \subseteq S_{\Upsilon}$. Then the following are equivalent:

(1) $\left\{s_{n}\right\}_{n \in \mathbb{N}}$ is $\Upsilon$-Cauchy.

(2) For each $\varepsilon>0$, we can find $N_{\varepsilon} \in \mathbb{N}$ such that $\Upsilon_{\sigma}\left(s_{n}, s_{m}, s_{m}\right)<\varepsilon$ for every $n, m \geq N_{\varepsilon}$ and $\sigma>0$.

(3) $\left\{s_{n}\right\}_{n \in \mathbb{N}}$ is a Cauchy sequence in the MM-space $\left(S, \theta_{\sigma}^{\Upsilon}\right)$.

Consider the GMM-space $(S, \Upsilon)$. Let the set of nonempty subsets, the set of nonempty finite subsets, and the set of nonempty compact of $\left(S, \mathcal{T}_{\Upsilon}\right)$ be denoted respectively by $\Gamma_{\mathcal{o}}(S)$, $\mathfrak{f}_{\mathrm{o}}(S)$, and $\mathfrak{R}_{\mathrm{o}}(S)$.

Proposition 2.10 ([3]) Let $(S, \Upsilon)$ be a GMM-space. Then $\Upsilon$ is a continuous function on $S \times S \times S \times \mathbb{J}^{0}$.

Let $T$ and $U$ be two (nonempty) subsets of a GMM-space $(S, \Upsilon)$.

For $s \in S$ and $\sigma>0$, let $\Upsilon_{\sigma}(s, T, U):=\inf \left\{\Upsilon_{\sigma}(s, t, u): t \in T, u \in U\right\}$.

Lemma 2.11 Let $(S, \Upsilon)$ be a GMM-space. Then, for each $s \in S, T, U \in \mathfrak{R}_{\mathrm{o}}(S)$ and $\sigma \in \mathbb{J}^{0}$, there are $t_{0} \in T, u_{0} \in U$ such that $\Upsilon_{\sigma}(s, T, U)=\Upsilon_{\sigma}\left(s, t_{0}, u_{0}\right)$.

Proof Let $s \in S, T, U \in \mathfrak{R}_{\mathrm{o}}(S)$ and $\sigma>0$. By Proposition 2.10, the functions $t \mapsto \Upsilon_{\sigma}(s, t, u)$, $u \mapsto \Upsilon_{\sigma}(s, t, u)$ are continuous. Thus, by compactness of $T$ and $U$, there exists $t_{0} \in T$, $u_{0} \in U$ such that $\inf _{t \in T, u \in U} \Upsilon_{\sigma}(s, t, u)=\Upsilon_{\sigma}\left(s, t_{0}, u_{0}\right)$, i.e., $\Upsilon_{\sigma}(s, T, U)=\Upsilon_{\sigma}\left(s, t_{0}, u_{0}\right)$.

Lemma 2.12 Let $(S, \Upsilon)$ be a GMM-space. Then, for each $s \in S$ and $T, U \in \mathfrak{R}_{\mathrm{o}}(S)$, the function $\sigma \mapsto \Upsilon_{\sigma}(s, T, U)$ is continuous on $\mathbb{J}^{0}$.

Proof The equality $\Upsilon_{\sigma}(s, T, U)=\inf _{t \in T, u \in U} \Upsilon_{\sigma}(s, t, u)$ and the continuity property of the function $\sigma \mapsto \Upsilon_{\sigma}(s, t, u)$ for each $t \in T$ and $u \in U$ on $\mathbb{J}^{0}$ imply the upper semi-continuity 
$\sigma \mapsto \Upsilon_{\sigma}(s, T, U)$ on $\mathbb{J}^{0}$. Consider $\sigma \in \mathbb{J}^{0}$, and let the sequence $\left(\sigma_{n}\right)_{n}$ in $\mathbb{J}^{0}$ converge to $\sigma$. Using Lemma 2.11 implies that we can find $t_{n} \in T$ and $u_{n} \in U$ for every $n \in \mathbb{N}$ such that $\Upsilon_{\sigma}(s, T, U)=\Upsilon_{\sigma_{n}}\left(s, t_{n}, u_{n}\right)$. From $T, U \in \mathfrak{R}_{\mathrm{o}}(S)$, we can find $\left(t_{n_{k}}\right)_{k}$ of $\left(t_{n}\right)_{n}\left(u_{n_{k}}\right)_{k}$ of $\left(u_{n}\right)_{n}$ and two points $t_{0} \in T$ and $u_{0} \in U$ such that $t_{n_{k}} \rightarrow t_{0}$ and $u_{n_{k}} \rightarrow u_{0}$ in $(S, \Upsilon)$. Hence $\lim _{k} \Upsilon_{\sigma_{n_{k}}}\left(s, t_{n_{k}}, u_{n_{k}}\right)=\Upsilon_{\sigma}\left(s, t_{0}, u_{0}\right)$ by Proposition 2.10, and thus $\lim _{k} \Upsilon_{\sigma_{n_{k}}}(s, T, U)=$ $\Upsilon_{\sigma}\left(s, t_{0}, u_{0}\right) \geq \Upsilon_{\sigma}(s, T, U)$. Consequently, $\sigma \mapsto \Upsilon_{\sigma}(s, T, U)$ is lower semi-continuous on $\mathbb{J}^{0}$.

Lemma 2.13 Consider the GMM-space $(S, \Upsilon)$. Then, for every $T \in \mathfrak{R}_{\mathrm{o}}(S), U, V \in \Gamma_{\mathrm{o}}(S)$ and $\sigma \in \mathbb{J}^{0}$, we can find $t_{0} \in T$ such that

$$
\sup \Upsilon_{\sigma}(T, U, V)=\Upsilon_{\sigma}\left(t_{0}, U, V\right)
$$

Proof Put $\delta=\sup _{t \in T} \Upsilon_{\sigma}(t, U, V)$. Then we can find a sequence $\left(t_{n}\right)_{n}$ in $T$ such that $\delta-\frac{1}{n}<$ $\Upsilon_{\sigma}\left(t_{n}, U, V\right)$ in which $n \in \mathbb{N}$. From $T \in \mathfrak{R}_{\mathcal{o}}(S)$, we can find a subsequence $\left(t_{n_{k}}\right)_{k}$ of $\left(t_{n}\right)_{n}$ and $t_{0} \in T$ such that $t_{n_{k}} \rightarrow t_{0}$ in $(S, \Upsilon)$.

Select $u \in U, v \in V$. According to Proposition 2.10,

$$
\lim _{k} \Upsilon_{\sigma}\left(t_{n_{k}}, u, v\right)=\Upsilon_{\sigma}\left(t_{0}, u, v\right)
$$

Since, for each $k \in \mathbb{N}, \delta-\frac{1}{n_{k}}<\Upsilon_{\sigma}\left(t_{n_{k}}, u, v\right)$, we get $\delta \leq \Upsilon_{\sigma}\left(t_{0}, u, v\right)$. We conclude that $\delta=$ $\Upsilon_{\sigma}\left(t_{0}, U, V\right)$.

Now, Lemmas 2.12 and 2.13 imply the next result.

Corollary 2.14 Consider the $G M M$-space $(S, \Upsilon)$. Assume that $T, U, V \in \mathfrak{R}_{\mathrm{o}}(S)$ and $\sigma \in \mathbb{J}^{0}$. Then we can find $t_{0} \in T, u_{0} \in U$, and $v_{0} \in V$ such that

$$
\sup _{t \in T} \Upsilon_{\sigma}(t, U, V)=\Upsilon_{\sigma}\left(t_{0}, u_{0}, v_{0}\right)
$$

Proposition 2.15 Consider the GMM-space $(S, \Upsilon)$. Then, for each $T, U, V \in \mathfrak{R}_{\mathrm{o}}(S)$, the function $\delta \mapsto \sup _{t \in T} \Upsilon_{\sigma}(t, U, V)$ is continuous on $\mathbb{J}^{0}$.

Proof It is easily proved by using Lemma 2.13, Lemma 2.12, and Proposition 2.10.

Remark 2.16 ([3]) Note that for $s, t, u \in S$ the function $0<\sigma \mapsto \Upsilon_{\sigma}(s, t, u) \in \mathbb{J}$ is nonincreasing on $\mathbb{J}^{0}$.

\section{GMM-Hausdorff distance on $\Re_{\mathrm{o}}(S)$}

Consider the GMM-space $(S, \Upsilon)$. We define a function $H_{\Upsilon}$ on $\mathfrak{R}_{\mathrm{o}}(S) \times \mathfrak{R}_{\mathrm{o}}(S) \times \mathfrak{R}_{\mathrm{o}}(S) \times \mathbb{J}^{0}$ by

$$
H_{\Upsilon}(T, U, V, \sigma)=\max \left\{\sup _{t \in T} \Upsilon_{\sigma}(t, U, V), \sup _{u \in U} \Upsilon_{\sigma}(T, u, V), \sup _{v \in V} \Upsilon_{\sigma}(T, U, v)\right\}
$$

for every $T, U, V \in \mathfrak{R}_{\mathfrak{o}}(S)$ and $\sigma \in \mathbb{J}^{0}$. 
Lemma 3.1 Consider the GMM-space $(S, \Upsilon), s \in S, T, U \in \mathfrak{R}_{\mathrm{o}}(S), V \in \Gamma_{\mathfrak{o}}(S)$, and $\alpha, \beta \in$ $\mathbb{J}^{0}$. Then

$$
\Upsilon_{\alpha+\beta}(s, T, V) \leq \Upsilon_{\alpha}(s, U, U)+\Upsilon_{\beta}\left(u_{s}, T, V\right)
$$

where $u_{s} \in U$ satisfies $\Upsilon_{\alpha}(s, U, U)=\Upsilon_{\alpha}\left(s, u_{s}, u_{s}\right)$.

Proof Using Lemma 2.11, for $u_{s} \in U$, we have $\Upsilon_{\alpha}(s, U, U)=\Upsilon_{\alpha}\left(s, u_{s}, u_{s}\right)$. Now, for each $t \in T, v \in V$, we have

$$
\Upsilon_{\alpha+\beta}(s, T, V) \leq \Upsilon_{\alpha+\beta}(s, t, v) \leq \Upsilon_{\alpha}\left(s, u_{s}, u_{s}\right)+\Upsilon_{\beta}\left(u_{s}, t, v\right)
$$

Then $\Upsilon_{\alpha+\beta}(s, T, V) \leq \Upsilon_{\alpha}(s, U, U)+\Upsilon_{\beta}\left(u_{s}, T, V\right)$.

Theorem 3.2 Consider the GMM-space $(S, \Upsilon)$. Then $\left(\Re_{\mathfrak{o}}(S), H_{\Upsilon}\right)$ is a GMM-space.

Proof Suppose that $T, U, V, W \in \mathfrak{R}_{\mathrm{o}}(S)$ and $\alpha, \beta \in \mathbb{J}^{0}$. By Lemma 2.13, there exist $t_{0} \in$ $T, u_{0} \in U$, and $v_{0} \in V$ such that $\sup _{t \in T} \Upsilon(t, U, V)=\Upsilon\left(t_{0}, U, V\right)$, $\sup _{u \in U} \Upsilon(T, u, V)=$ $\Upsilon\left(T, u_{0}, V\right)$, and $\sup _{v \in V} \Upsilon(T, U, v)=\Upsilon\left(T, U, v_{0}\right)$.

Then $H_{\Upsilon}(T, U, V, \alpha) \geq 0$. Furthermore, it is obvious that

$$
T=U=V \Leftrightarrow H_{\Upsilon}(T, U, V, \alpha)=0 .
$$

Now, according to Lemma 3.1, we have

$$
\sup _{t \in T} \Upsilon_{\alpha+\beta}(t, U, W) \leq \sup _{t \in T} \Upsilon_{\alpha}(t, V, V)+\sup _{t \in T} \Upsilon_{\beta}\left(v_{t}, U, W\right)
$$

Since $\left\{v_{t}: t \in T\right\} \subseteq V$, $\sup _{t \in T} \Upsilon_{\beta}\left(v_{t}, U, W\right) \leq \sup _{v \in V} \Upsilon_{\beta}(v, U, W)$, so

$$
\sup _{t \in T} \Upsilon_{\alpha+\beta}(t, U, W) \leq \sup _{t \in T} \Upsilon_{\alpha}(t, V, V)+\sup _{v \in V} \Upsilon_{\beta}(\nu, U, W)
$$

In the same way, we obtain

$$
\begin{aligned}
& \sup _{u \in U} \Upsilon_{\alpha+\beta}(T, u, W) \leq \sup _{u \in U} \Upsilon_{\alpha}(u, V, V)+\sup _{v \in V} \Upsilon_{\beta}(v, T, W), \\
& \sup _{w \in W} \Upsilon_{\alpha+\beta}(T, U, w) \leq \sup _{w \in W} \Upsilon_{\alpha}(w, V, V)+\sup _{v \in V} \Upsilon_{\beta}(v, T, W) .
\end{aligned}
$$

Then, it easily follows that

$$
H_{\Upsilon}(T, U, W, \alpha+\beta) \leq H_{\Upsilon}(T, V, V, \alpha)+H_{\Upsilon}(V, U, W, \beta) .
$$

By Proposition 2.15, we conclude that $\alpha \mapsto H_{\Upsilon}(T, U, V, \alpha)$ is continuous on $\mathbb{J}^{0}$.

Then $\left(\mathfrak{R}_{\mathrm{o}}(S), H_{\Upsilon}\right)$ is a GMM-space. 


\section{$4 \Upsilon$-Cauchy sequences in a GMM-space}

In this section we study $\Upsilon$-Cauchy sequences in a GMM-space.

Lemma 4.1 Consider the GMM-space $(S, \Upsilon)$. For each $\mu \in \mathbb{J}^{0}$, define a function $F_{\mu, \Upsilon}(s, t$, $u)=\inf \left\{\sigma>0, \Upsilon_{\sigma}(s, t, u)<\mu\right\}$ for any $s, t, u \in S$. Then

(i) For any $\lambda \in \mathbb{J}^{0}$, we can find $\mu \in \mathbb{J}^{0}$ such that

$$
F_{\lambda, \Upsilon}\left(s_{0}, s_{m}, s_{m}\right) \leq \sum_{i=0}^{m-1} F_{\mu, \Upsilon}\left(s_{i}, s_{i+1}, s_{i+1}\right)
$$

for all $s_{0}, s_{1}, \ldots, s_{m} \in S$.

(ii) Let $\left\{s_{n}\right\}_{n}$ be a convergent sequence in a GMM-space $(S, \Upsilon)$, then we have $F_{\lambda, \Upsilon}\left(s, s_{n}\right.$, $\left.s_{n}\right) \rightarrow 0$ and vice versa.

Proof (i) For every $\lambda \in \mathbb{J}^{0}$, we can find $\mu \in \mathbb{J}^{0}$ such that $m \mu<\lambda$. For any given $m \in \mathbb{Z}^{+}$, we put

$$
F_{\mu, \Upsilon}\left(s_{i}, s_{i+1}, s_{i+1}\right)=\sigma_{i}
$$

for $i=0,1,2, \ldots, m-1$.

For every $\varepsilon>0$, it is obvious that $F_{\mu, \Upsilon}\left(s_{i}, s_{i+1}, s_{i+1}\right)<\sigma_{i}+\frac{\varepsilon}{m}$, in which $i=0,1, \ldots, m-1$. Then $\Upsilon_{\sigma_{i}+\frac{\varepsilon}{m}}\left(s_{i}, s_{i+1}, s_{i+1}\right)<\mu$ for $i=0,1, \ldots, m-1$. Now, using (GMM-5), we get

$$
\begin{aligned}
& \Upsilon_{\sigma_{0}+\sigma_{1}+\cdots+\sigma_{m-1+\varepsilon}}\left(s_{0}, s_{m}, s_{m}\right) \\
& \quad \leq \Upsilon_{\sigma_{0}+\frac{\varepsilon}{m}}\left(s_{0}, s_{1}, s_{1}\right)+\cdots+\Upsilon_{\sigma_{m-1}+\frac{\varepsilon}{m}}\left(s_{m-1}, s_{m}, s_{m}\right) \\
& \quad<\underbrace{\mu+\cdots+\mu}_{m}<\lambda,
\end{aligned}
$$

which implies that

$$
F_{\lambda, \Upsilon}\left(s_{0}, s_{m}, s_{m}\right) \leq \sigma_{0}+\sigma_{1}+\cdots+\sigma_{m-1}+\varepsilon .
$$

Using (4.2) we get

$$
F_{\lambda, \Upsilon}\left(s_{0}, s_{m}, s_{m}\right) \leq \sum_{i=0}^{m-1} F_{\mu, \Upsilon}\left(s_{i}, s_{i+1}, s_{i+1}\right)+\varepsilon
$$

for all $s_{0}, s_{1}, \ldots, s_{m} \in S$. Tending $\varepsilon$ to 0 in (4.4) implies that (4.1).

(ii) We have $\Upsilon_{\eta}\left(s, s_{n}, s_{n}\right)<\lambda \Leftrightarrow F_{\lambda, \Upsilon}\left(s, s_{n}, s_{n}\right)<\eta$ for every $\eta>0$.

Lemma 4.2 Consider the GMM-space $(S, \Upsilon)$. If $\Upsilon_{\sigma}(s, t, u)=C$ for every $s, t, u \in S$ and $\sigma \in$ $\mathbb{J}^{0}$, then

$$
C=0 .
$$


Proof Putting $s=t=u$ in (4.5), we get $C=0$.

Here, we consider a class of mappings $\phi: \mathbb{J}^{0} \rightarrow \mathbb{J}^{0}$ which are onto, strictly increasing, and $\phi(\sigma)<\sigma$ for all $\sigma \in \mathbb{J}^{0}$.

Lemma 4.3 Consider the GMM-space $(S, \Upsilon)$. Then

$$
\inf \left\{\phi^{n}(\sigma)>0: \Upsilon_{\sigma}(s, t, u)<\lambda\right\} \leq \phi^{n}\left(\inf \left\{\sigma>0: \Upsilon_{\sigma}(s, t, u)<\lambda\right\}\right)
$$

for each $s, t, u \in S, \lambda \in \mathbb{J}^{0}$, and $n \in \mathbb{N}$.

Proof Fix $\sigma \in \mathbb{J}^{0}$ with $\Upsilon_{\sigma}(s, t, u)<\lambda$. Then $\phi^{n}(\sigma) \in \mathbb{J}^{0}$. Also $\phi^{n}(\sigma) \geq \inf \left\{\phi^{n}(\delta)>0\right.$ : $\left.\Upsilon_{\delta}(s, t, u)<\lambda\right\}$, and so we have

$$
\sigma \geq\left(\phi^{n}\right)^{-1}\left(\inf \left\{\phi^{n}(\delta)>0: \Upsilon_{\delta}(s, t, u)<\lambda\right\}\right)
$$

Then

$$
\inf \left\{\sigma>0, \Upsilon_{\sigma}(s, t, u)<\lambda\right\} \geq\left(\phi^{n}\right)^{-1}\left(\inf \left\{\phi^{n}(\delta)>0: \Upsilon_{\delta}(s, t, u)<\lambda\right\}\right)
$$

and we conclude that

$$
\inf \left\{\phi^{n}(\sigma)>0: \Upsilon_{\sigma}(s, t, u)<\lambda\right\} \leq \phi^{n}\left(\inf \left\{\sigma>0: \Upsilon_{\sigma}(s, t, u)<\lambda\right\}\right)
$$

Lemma 4.4 Consider the GMM-space $(S, \Upsilon)$. Suppose that $\left\{s_{n}\right\} \subseteq S$ such that $\Upsilon_{\phi^{n}(\sigma)}\left(s_{n}\right.$, $\left.s_{n+1}, s_{n+1}\right) \leq \Upsilon_{\sigma}\left(s_{0}, s_{1}, s_{1}\right)$ for all $\sigma \in \mathbb{J}^{0}$. Then $\left\{s_{n}\right\}$ is a $\Upsilon$-Cauchy sequence.

Proof Using Lemma 4.3, we get

$$
\begin{aligned}
F_{\mu, \Upsilon}\left(s_{n}, s_{n+1}, s_{n+1}\right) & =\inf \left\{\phi^{n}(\sigma)>0: \Upsilon_{\phi^{n}(\sigma)}\left(s_{n}, s_{n+1}, s_{n+1}\right)<\mu\right\} \\
& \leq \inf \left\{\phi^{n}(\sigma)>0: \Upsilon_{\sigma}\left(s_{0}, s_{1}, s_{1}\right)<\mu\right\} \\
& \leq \phi^{n}\left(\inf \left\{\sigma>0: \Upsilon_{\sigma}\left(s_{0}, s_{1}, s_{1}\right)<\mu\right\}\right) \\
& =\phi^{n}\left(F_{\mu, \Upsilon}\left(s_{0}, s_{1}, s_{1}\right)\right)
\end{aligned}
$$

for every $\mu \in \mathbb{J}^{0}$.

For every $\lambda \in \mathbb{J}^{0}$, there exists $\theta \in \mathbb{J}^{0}$ such that

$$
\begin{aligned}
F_{\lambda, \Upsilon}\left(s_{n}, s_{m}, s_{m}\right) \leq & F_{\theta, \Upsilon}\left(s_{m-1}, s_{m}, s_{m}\right)+F_{\theta, \Upsilon}\left(s_{m-2}, s_{m-1}, s_{m-1}\right)+\cdots \\
& +F_{\theta, \Upsilon}\left(s_{n}, s_{n+1}, s_{n+1}\right) \\
\leq & \sum_{i=n}^{m-1} \phi^{i}\left(F_{\theta, \Upsilon}\left(s_{0}, s_{1}, s_{1}\right)\right) \rightarrow 0 .
\end{aligned}
$$

By Lemma 4.1, $\left\{s_{n}\right\}$ is a $\Upsilon$-Cauchy sequence. 


\section{GMM-fractal spaces}

Hutchinson considered the concept of fractal theory by studying the iterated function system (IFS) [12]. This subject was generalized by Barnsley [4], Bisht [5], Imdad [13], and Ri [14].

Definition 5.1 Consider the GMM-space $(S, \Upsilon)$. A mapping $\Omega: S \rightarrow S$ is said to be a GMM- $\phi$-contractive mapping if $\Upsilon_{\phi(\sigma)}(\Omega(s), \Omega(t), \Omega(u)) \leq \Upsilon_{\sigma}(s, t, u)$ for every $s, t, u \in S$ and $\sigma \in \mathbb{J}^{0}$.

Definition 5.2 A GMM iterated function system (shortly, GMMIFS) is a finite set of GMM- $\phi$-contractions $\left\{\Omega_{1}, \Omega_{2}, \ldots, \Omega_{m}\right\},(m \geq 2)$ that is defined on a complete GMM-space $(S, \Upsilon)$.

For a GMMIFS, we can find a unique nonempty compact set $\Gamma$ of the complete GMMspace $(S, \Upsilon)$ in which $\Gamma=\bigcup_{i=1}^{m} \Omega_{i}(\Gamma)$ and $\Gamma$ is a fractal set called the attractor of the respective (GMMIFS). In this case, the corresponding attractor GMMIFS is said to be GMMfractal space.

Lemma 5.3 Consider the GMM-space $(S, \Upsilon)$. Assume that $\Omega: S \rightarrow S$ is a mapping such that

$$
\Upsilon_{\phi(\sigma)}(\Omega(s), \Omega(t), \Omega(u)) \leq \Upsilon_{\sigma}(s, t, u)
$$

for every $s, t, u \in S$ and $\sigma \in \mathbb{J}^{0}$. Then the sequence $\left\{\Omega^{n}(s)\right\}_{n=1}^{\infty}$ is GMMCS.

Proof Assume that $\left\{s_{n}: \Omega^{n}(s)\right\}_{n=1}^{+\infty}$. $\left\{s_{n}\right\}$ is a sequence satisfying the conditions of Lemma 4.4. By using the induction, we have

$$
\Upsilon_{\sigma}(s, \Omega(s), \Omega(s)) \leq \Upsilon_{\sigma}(s, \Omega(s), \Omega(s))
$$

if

$$
\Upsilon_{\phi^{n}(\sigma)}\left(\Omega^{n}(s), \Omega^{n+1}(s), \Omega^{n+1}(s)\right) \leq \Upsilon_{\sigma}(s, \Omega(s), \Omega(s)),
$$

then

$$
\begin{aligned}
& \Upsilon_{\phi^{n+1}(\sigma)}\left(\Omega^{n+1}(s), \Omega^{n+2}(s), \Omega^{n+2}(s)\right)=\Upsilon_{\phi\left(\phi^{n}(\sigma)\right)}\left(\Omega\left(\Omega^{n}(s), \Omega\left(\Omega^{n+1}(s)\right)\right),\right. \\
& \Omega\left(\Omega^{n+1}(s)\right) \leq \Upsilon_{\phi^{n}(\sigma)}\left(\Omega^{n}(s), \Omega^{n+1}(s), \Omega^{n+1}(s)\right) \leq \Upsilon_{\sigma}(s, \Omega(s), \Omega(s)) .
\end{aligned}
$$

Therefore,

$$
\Upsilon_{\phi^{n}(\sigma)}\left(s_{n}, s_{n+1}, s_{n+1}\right) \leq \Upsilon_{\sigma}\left(s_{0}, s_{1}, s_{1}\right)
$$

hence $\left\{s_{n}=\Omega^{n}(s)\right\}_{n=1}^{\infty}$ is a GMMCS.

Lemma 5.4 Consider the GMM-space $(S, \Upsilon)$ and a GMM- $\phi$-contractive map $\Omega$ such that

$$
\Upsilon_{\phi(\sigma)}(\Omega(s), \Omega(t), \Omega(u)) \leq \Upsilon_{\sigma}(s, t, u)
$$

for every $s, t, u \in S$ and $\sigma \in \mathbb{J}^{0}$. Then $\Omega$ has a unique fixed point $\alpha$ in $S$. 
Proof Using Lemma 5.3 and (5.1), we get the sequence $\left\{\Omega^{n}(s)\right\}_{n=1}^{+\infty}$ is GMMCS for each $s \in S$ and $\lim _{n \rightarrow \infty} \Omega^{n}(s)=\alpha \in S$.

Letting $s_{0}=s$ and $s_{n}=\Omega^{n}(s)$ for each $n \geq 1$, since $\lim _{n \rightarrow \infty} \Omega^{n}(s)=\alpha$, we have $\lim \Upsilon_{\sigma}\left(s_{n}, \alpha\right.$, $\alpha)=0$ for each $\sigma \in \mathbb{J}^{0}$.

On the other hand, we recognize

$$
\Upsilon_{\phi(\sigma)}\left(\Omega(\alpha), s_{n+1}, s_{n+1}\right) \leq \Upsilon_{\sigma}\left(\alpha, s_{n}, s_{n}\right)
$$

for each $n \in \mathbb{N}$ and each $\sigma>0$. Then

$$
\begin{aligned}
\Upsilon_{\phi(\sigma)}(\Omega(\alpha), \alpha, \alpha) & =\lim _{n \rightarrow \infty} \Upsilon_{\phi(\sigma)}\left(\Omega(\alpha), s_{n+1}, s_{n+1}\right) \\
& \leq \lim _{n \rightarrow \infty} \Upsilon_{\sigma}\left(\alpha, s_{n}, s_{n}\right)=0
\end{aligned}
$$

for each $\sigma>0$. Therefore, $\alpha=\Omega(\alpha)$, and $\alpha$ is a fixed point of $\Omega$.

Now, we have to prove that $\alpha$ is the unique fixed point of $\Omega$. If $\beta$ is another fixed point of $\Omega$, then for any $\sigma \in \mathbb{J}^{0}$

$$
\Upsilon_{\sigma}(\alpha, \alpha, \beta)=\Upsilon_{\sigma}(\Omega(\alpha), \Omega(\alpha), \Omega(\beta)) \geq \Upsilon_{\phi(\sigma)}(\Omega(\alpha), \Omega(\alpha), \Omega(\beta))
$$

On the other hand, since $\Upsilon_{\sigma}(s, t, t)$ is nonincreasing and $\phi(\sigma)<\sigma$, we have

$$
\Upsilon_{\phi(\sigma)}(\Omega(\alpha), \Omega(\alpha), \Omega(\beta)) \geq \Upsilon_{\sigma}(\Omega(\alpha), \Omega(\alpha), \Omega(\beta))=\Upsilon_{\sigma}(\alpha, \alpha, \beta)
$$

Hence $\Upsilon_{\sigma}(\alpha, \alpha, \beta)=C$ for all $\sigma \in \mathbb{J}^{0}$. From Lemma 4.2 we get $C=0$. Therefore, $\alpha=\beta$, i.e., $\alpha$ is a unique fixed point of $\Omega$.

\section{Concluding remarks}

In this paper, we studied some topological properties of Hausdorff distance on generalized modular metric and could define a generalized modular fractal space.

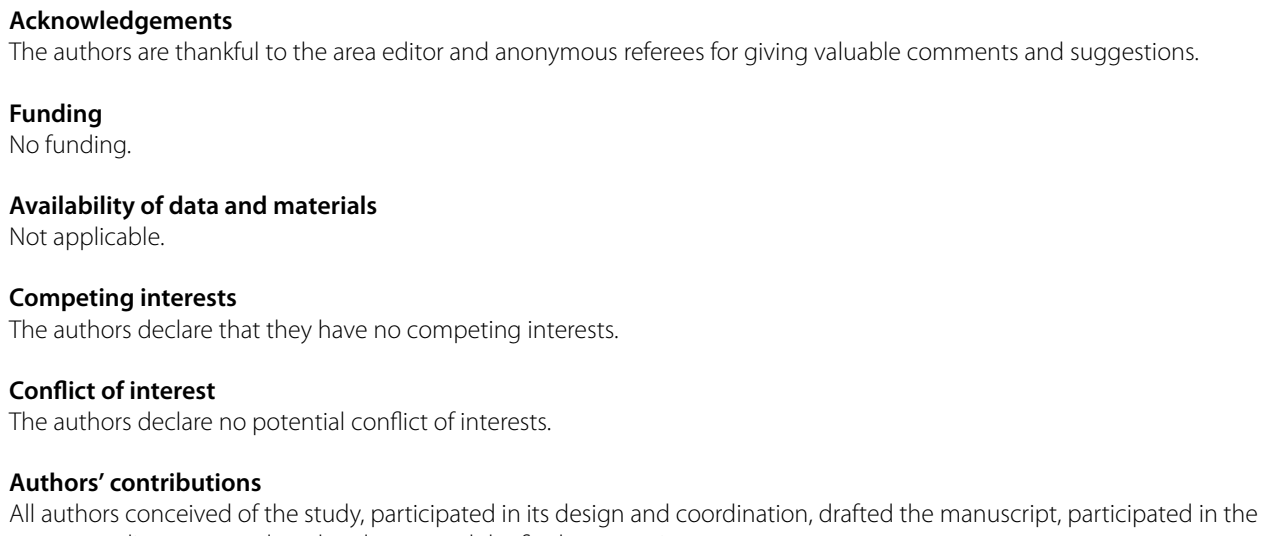

\section{Publisher's Note}

Springer Nature remains neutral with regard to jurisdictional claims in published maps and institutional affiliations. 
References

1. Abdou, A.A.N.: Some fixed point theorems in modular metric spaces. J. Nonlinear Sci. Appl. 9(6), 4381-4387 (2016)

2. Abdou, A.A.N.: Fixed points of Kamnan maps in modular metric spaces. AIMS Math. 5(6), 6395-6403 (2020)

3. Azadifar, B., Maramaei, M., Sadeghi, G.: On the modular G-metric spaces and fixed point theorems. J. Nonlinear Sci. Appl. 6, 293-304 (2013)

4. Barnsley, M.: Fractals Everywhere. Academic Press, Boston (1988)

5. Bisht, R.K.: Comment on: A new fixed point theorem in the fractals space. Indag. Math. 29(2), 819-823 (2018)

6. Chistyakov, V.V.: Modular metric spaces generated by F-modulars. Folia Math. 14, 3-25 (2008)

7. Chistyakov, V.V.: Modular metric spaces I basic concepts. Nonlinear Anal. 72, 1-14 (2010)

8. Chistyakov, V.V.: Metric Modular Spaces. Theory and Applications. Springer Briefs in Mathematics. Springer, Cham (2015)

9. Cho, Y.J., Saadati, R., Sadeghi, G.: Quasi-contractive mappings in modular metric spaces. J. Appl. Math. 2012, Article ID 907951 (2012)

10. Ege, O., Park, C., Ansari, A.H.: A different approach to complex valued Gb-metric spaces. Adv. Differ. Equ. 2020, Article ID $152(2020)$

11. Gholidahneh, A., Sadeghi, Sh., Ege, O., Mitrovic, Z.D., De la Sen, M.: The Meir-Keeler type contractions in extended modular b-metric spaces with an application. AIMS Math. 6(2), 1781-1799 (2021)

12. Hutchison, J.E.: Fractals and self-similarity. Indiana Univ. Math. J. 30(5), 713-747 (1981)

13. Imdad, M., Alfaqih, W.M., Khan, I.A.: Weak $\theta$-contractions and some fixed point results with applications to fractal theory. Adv. Differ. Equ. 2018, Article ID 439 (2018)

14. Ri, S.il A.: A new fixed point theorem in the fractal space. Indag. Math. 27(1), 85-93 (2016)

\section{Submit your manuscript to a SpringerOpen ${ }^{\circ}$ journal and benefit from:}

- Convenient online submission

Rigorous peer review

- Open access: articles freely available online

- High visibility within the field

- Retaining the copyright to your article

Submit your next manuscript at $\gg$ springeropen.com 